\section{Human equilibrative nucleoside transporter 1 and carcinoma of the ampulla of Vater: expression differences in tumour histotypes}

\author{
G. Perrone, ${ }^{1}$ S. Morini, ${ }^{2}$ D. Santini, ${ }^{3}$ \\ C. Rabitti, ${ }^{1}$ B. Vincenzi, ${ }^{3}$ R. Alloni, ${ }^{4}$ \\ A. Antinori, ${ }^{5}$ P. Magistrelli, ${ }^{5}$ R. Lai, ${ }^{6}$ \\ C. Cass, ${ }^{6}$ J.R. Mackey, ${ }^{6}$ R. Coppola, ${ }^{4}$ \\ G. Tonini, ${ }^{3}$ A. Onetti Muda'
}

'Department of Anatomical Pathology; 'Biomedical Research; ${ }^{3}$ Medical Oncology; ${ }^{4}$ Surgery, Campus Bio-Medico University, Rome, Italy; ${ }^{5}$ Department of Surgery, Catholic University of the Sacred Heart, Rome, Italy; ${ }^{6}$ Cross Cancer Institute and Department of Oncology, University of Alberta, Edmonton, Alberta, Canada

\section{Abstract}

The human equilibrative nucleoside transporter 1 (hENT1) is the major means by which gemcitabine enters human cells; recent evidence exists that hENT1 is expressed in carcinoma of the ampulla of Vater and that it should be considered as a molecular prognostic marker for patients with resected ampullary cancer. Aim of the present study is to evaluate the variations of hENT1 expression in ampullary carcinomas and to correlate such variations with histological subtypes and clinicopathological parameters. Forty-one ampullary carcinomas were histologically classified into intestinal, pancreaticobiliary and unusual types. hENT1 and Ki67 expression were evaluated by immunohistochemistry, and apoptotic cells were identified by the terminal deoxynucleotidyl transferase mediated deoxyuridine triphosphate biotin nick end labelling (TUNEL) method. hENT1 overexpression was detected in $63.4 \%$ ampullary carcinomas. A significant difference in terms of hENT1 and Ki67 expression was found between intestinal vs. pancreaticobiliary types $(\mathrm{P}=0.03$ and $\mathrm{P}=0.009$ respectively). Moreover, a significant statistical positive correlation was found between apoptotic and proliferative Index $(\mathrm{P}=0.036)$, while no significant correlation was found between hENT1 and apoptosis. Our results on hENT1 expression suggest that classification of ampullary carcinoma by morphological subtypes may represent an additional tool in prospective clinical trials aimed at examining treatment efficacy; in addition, data obtained from Ki67 and TUNEL suggest a key role of hENT1 in tumour growth of ampullary carcinoma.

\section{Introduction}

The ampulla of Vater consists of papilla, common channel, distal common bile duct (ampullo-biliary portion), and the distal main pancreatic duct (ampullo-pancreatical portion). The papilla is covered by intestinal mucosa, while the inner parts of the ampulla are lined by a simple mucinous epithelium similar to that of the pancreaticobiliary tree., ${ }^{1,2}$ Therefore, ampullary carcinomas may arise from two different types of mucosa, which might reflect the broad histomorphological spectrum of these tumors. ${ }^{3}$ Kimura et al., for the first time, have distinguished pancreaticobiliary type and intestinal type of ampullary carcinoma. ${ }^{2}$ In 2000, Albores-Saavedra et al. defined the pancreaticobiliary and intestinal types as main types and added the so-called "unusual types". 4

The adjuvant therapy for resected ampullary carcinoma is poorly studied due, in part, to the rarity of the cancer. Gemcitabine (Gemzar, Eli Lilly, Indianapolis, In, USA) is a pyrimidine nucleoside analogue that is the most single effective agent in the chemotherapy of pancreatic adenocarcinoma ${ }^{5}$ and appears to be a reasonable alternative to best supportive care in the treatment of advanced biliary tract cancers, on the basis of encouraging results in non-randomized clinical studies. ${ }^{6,7}$ The human equilibrative nucleoside transporter 1 (hENT1) is an ubiquitous specialized plasma membrane nucleoside transporters protein and it represents the major means by which gemcitabine enters human cells. ${ }^{8,9}$ Tissue expression of hENT1 protein can be evaluated using immunohistochemistry; by this method, its amount and distribution has been assessed in a number of malignant and benign tissues. ${ }^{10-14}$ In a recent report, we demonstrated that hENT1 is expressed in carcinomas of the ampulla of Vater and should be considered a molecular prognostic marker for patients with resected ampullary cancer. ${ }^{15}$ Moreover, in a recent prospective randomized trial, hENT1 protein expression was associated with increased overall survival and disease-free survival in pancreatic cancer patients who received gemcitabine. ${ }^{16}$ Given the high variability of histological presentation of ampullary carcinomas, we aimed at carefully assessing hENT1 expression in the different histotypes of the tumour, as well as its relationship with the proliferation and apoptosis indices (respectively PI and AI). This was performed by means of immunohistochemistry and TUNEL methods on surgical specimens from radically resected ampullary carcinomas.
Correspondence: Giuseppe Perrone, Department of Anatomical Pathology, Campus Bio-Medico University, via Álvaro del Portillo 200, 00128 Rome, Italy.

Tel. +39.06.225411155 - Fax: +39.06 .225411929 . E-mail: g.perrone@unicampus.it

Key words: human equilibrative nucleoside transporter 1, histotypes, vater ampulla, cancer, immunohistochemistry.

Acknowledgments: the financial support for this study was received from Università Campus BioMedico di Roma (Grant: "Progetto d'Ateneo 2008") and "Consorzio Interuniversitario Trapianti d'organo", Rome, Italy.

Received for publication: 29 April 2010 Accepted for publication: 19 July 2010.

This work is licensed under a Creative Commons Attribution 3.0 License (by-nc 3.0).

(C) Copyright G. Perrone et al., 2010

Licensee PAGEPress, Italy

European Journal of Histochemistry 2010; 54:e38 doi:10.4081/ejh.2010.e38

\section{Materials and Methods}

\section{Specimen acquisition and histologi- cal examination}

To be eligible for the study, each subject underwent surgical resection for tumours of ampullary origin with curative intent, and only patients without known residual disease were analyzed. The procedures followed were in accordance with the ethical standards of the local responsible committee on human experimentation (Campus Bio-Medico University, Rome, Italy). All specimens underwent gross anatomical examination according to the procedure described by Rosai including evaluation of all anatomic structures (pancreatic duct, ampulla of Vater, common bile duct, pancreatic head).$^{17}$ All tumours included in the study were limited to the ampulla, or were primarily located in the ampulla with secondarily spreading into the neighbouring structures. TNM (tumour local extension, nodes and metastasis status) classification was reassessed following the International Union Against Cancer criteria. ${ }^{18}$ Histological slides were independently re-examined by two pathologists and all tumours were classified histologically as intestinal-type, pancreaticobiliary-type and unusual-type. ${ }^{4}$ Agreement in histological evaluation between the two observers was $>90 \%$. In cases of disagreement, a final decision was determined by consensus after re-examination. 


\section{Immunohistochemistry}

The specimens were fixed in $4 \%$ neutralbuffered formaldehyde and routinely embedded in paraffin. Based on the quality of the morphologic preservation of all available haematoxylin and eosin stained slides of the surgical specimen sections, we selected one paraffin block for each case. Immunohistochemical staining was performed as previously described. ${ }^{16}$ Briefly, representative tumour blocks were sectioned at $3 \mu \mathrm{m}$ and processed by the streptoavidin-biotin method. Incubation was performed in a humidified chamber overnight at $4^{\circ} \mathrm{C}$ with anti-hENT1 mouse monoclonal antibody (dilution: $10 \mu \mathrm{g} / \mathrm{mL}$ ), developed and characterized as previously described, ${ }^{10}$ and with anti-Ki-67 mouse mAb (1:50, clone MIB-1, DAK0, Cytomation Inc., Carpinteria, CA, USA). Sections were then incubated with goat antimouse dextran conjugate (DAKO Envision, DAKO, Cytomation Inc., Carpinteria, CA, USA) for 30 min. 3-3'-diaminobenzidine (DAB, DAKO, Cytomation Inc., Carpinteria, CA, USA) was used as chromogen. Negative control slides processed without primary antibody were included for each staining.

Scoring for hENT1 was based on the relative staining intensity of the ampullary tumour when compared to normal membrane hENT1 staining of cells of the islets of Langerhans and lymphocytes, which served as internal control. Staining intensity was graded as absent (0), positive, less intense than internal control $(1+)$, positive, same intensity as internal control $(2+)$, positive, more intense than internal control (3+). In samples with varying staining intensities of hENT1, the percentages of each staining intensity were recorded. hENT1 expression was considered "significant" when staining intensity of $2+$ and $3+$ was present in the majority of tumour tissue ( $\geq 50 \%$ of the tumour cells). Ki-67 staining was evaluated by counting the number of positively stained nuclei in at least 1000 tumour cells; immunoreactivity was graded by the percentage of nuclear staining (defined as proliferative index).

\section{Detection of apoptosis}

Apoptotic cells were identified by the TUNEL method using the ApopTag TUNEL kit (Oncor, Gaithersburg, MD, USA) according to the manufacturer's instructions. Dewaxed and rehydrated specimens were incubated in proteinase $\mathrm{K}(40 \mathrm{mg} / \mathrm{mL})$ for one hour at $37^{\circ} \mathrm{C}$, and were then treated with $3 \% \mathrm{H}_{2} \mathrm{O}_{2}$ in methanol for $30 \mathrm{~min}$ at room temperature. After adding equilibration buffer for $5 \mathrm{~min}$ at room temperature, the terminal deoxynucleotidyl transferase enzyme was pipetted onto the sections and incubated at $37^{\circ} \mathrm{C}$ for 2 hours. The reaction was stopped by incubating the sections in stop buffer for $30 \mathrm{~min}$ at $37^{\circ} \mathrm{C}$. Anti-digoxigenin peroxidase was added to the slides, followed by incubation for $30 \mathrm{~min}$ at $37^{\circ} \mathrm{C}$. Slides were stained with diaminobenzine for $10 \mathrm{~min}$ and counterstained with haematoxylin. The number of apoptotic bodies was expressed in relation to the total number of cells within at least 1000 tumour cells counted in high-power fields. Strict criteria were used to define a cell as apoptotic on TUNEL stained sections, i.e. the presence of a condensed and often fragmented nucleus together with a surrounding halo. Cells containing weakly to moderate TUNEL positive nuclei in the absence of these additional morphological features were not assessed as apoptotic. The AI was calculated as the percentage of tumour cell apoptotic bodies per total number of tumour cells counted for each case. Tumour specimens was stratified, according to $\mathrm{AI}$, into $\leq 10 \%$ or $>10 \% .^{19}$

\section{Statistical analysis}

Kruskal-Wallis test was used to establish the difference for nonparametric independent variables, and was applied to establish differences among the ampullary histotypes for: tumour local extension (T factor), nodal status ( $\mathrm{N}$ factor), differentiation grade and hENT1 expression. Mann-Whitney test was used to establish the difference for two independent samples, and was used to verify differences in terms of hENT1 expression and PI between pancreaticobiliary vs. intestinal or unusual types, and between intestinal vs. unusual type. Furthermore, Mann-Whitney test was applied to establish the relationship between hENT1 and AI. P values less than 0.05 were regarded as statistically significant in two-tailed tests.

Table 1. Patients' characteristics.

\begin{tabular}{lc}
\hline Total number & 41 \\
Median age (range) & $63(38-78)$ years \\
\hline Gender & \\
Male vs. female & 23 vs. $18(56.1 \%$ vs. $43.9 \%)$ \\
T factor & $7(17.1 \%)$ \\
T1 & $17(41.5 \%)$ \\
T2 & $15(36.6 \%)$ \\
T3 & $2(4.9 \%)$ \\
T4 & \\
\hline N factor & $23(56.1 \%)$ \\
Negative & $18(43.9 \%)$ \\
Positive & \\
Grade & $13(31.7 \%)$ \\
Well differentiated & $21(51.2 \%)$ \\
Moderate differentiated & $7(17.1 \%)$ \\
Poor differentiated & \\
\hline Histotypes & $20(48.8 \%)$ \\
Intestinal & $14(34.1 \%)$ \\
Pancreaticobiliary & $7(17.1 \%)$ \\
Unusual & \\
\hline
\end{tabular}

SPSS software (version 14.0.1, 2005, SPSS Inc.) was used for statistical analysis.

\section{Results}

\section{Patients' characteristics}

The main clinicopathological features are summarized in Table 1. The cohort consisted of 41 patients with pathological diagnosis of radically resected cancer of the ampulla (23 nosis was 63 years (range 38-78). Seven patients (17.1\%) were classified as T1, 17 (41.5\%) as T2, 15 (36.6\%) as T3, and 2 (4.9\%) as T4. Eighteen (43.9\%) patients had locoregional lymph node metastasis. A positive statistical correlation was found between $\mathrm{T}$ and $\mathrm{N}$ factor $(r=0.507 ; \mathrm{P}=0.001)$.

According to histological criteria published by Albores-Saavedra, ${ }^{4}$ we found 20 (48.8\%) intestinal-type carcinomas, 14 (34.1\%) pancreaticobiliary-type carcinomas, and 7 (17.1\%) unusual-type carcinomas (4 undifferentiated, 1 signet ring, 1 clear cell and 1 small cell carcinoma). A significant difference in the differentiation grade was found among the histotypes $(\mathrm{P}=0.007)$. In particular, significant differences were found between unusual-type vs. intestinal and pancreaticobiliary $(\mathrm{P}=0.001$ and $\mathrm{P}=0.003$, respectively), while no difference was found between intestinal vs. pancreaticobiliary $(\mathrm{P}=0.888)$. These results are consistent with the histological classification since "unusual type" is a subgroup including poorly differentiated ampullary cancers. No significant difference was found in $\mathrm{T}$ and $\mathrm{N}$ factors among ampullary carcinoma subtypes (Table 2). men and 18 women). The median age at diag- 
Human equilibrative nucleoside transporter 1 immunostaing and apoptosis indices

hENT1 immunostaining was normally observed in the cells of the islets of Langerhans and lymphocytes, and used as internal positive control. Tumour cells displayed hENT1 staining localized both to the cytoplasm and to the plasma membrane; peritumoral stromal cells were constantly negative (Figure 1), while normal intestinal epithelium showed focal and weak positivity in the Lieberkuhn crypts (data not shown). Among the 41 tissue samples, 26 (63.4\%) showed significant hENT1 expression (intensity scores of $2+$ and/or $3+$ in more than $60 \%$ of tumour tissue). AI was $>10 \%$ in 12 (29.3\%) ampullary cancer specimens.

\section{Human equilibrative nucleoside transporter 1 and morphological histotypes}

Significant hENT1 expression was evident in 17/20 (85\%) intestinal, 7/14 (50\%) pancreaticobiliary and $2 / 7$ (28.6\%) unusual carcinomas. A significant statistical difference in hENT1 expression was found among the histotypes $(\mathrm{P}=0.014)$. In particular, a significant difference was found between intestinal vs. pancreaticobiliary types and between intestinal vs. unusual types; no difference was found between pancreaticobiliary vs. unusual types (Table 3).

\section{Proliferation indices, apoptosis indices and others variables}

In terms of PI, a significant statistical difference was found between intestinal (median 40\%; Inter Quartile Range [I.Q.R] 30-42.5\%) and pancreaticobiliary histotypes (median 25\%; I.Q.R. 6.25-40\%) ( $\mathrm{P}=0.009)$. No difference was found between unusual-types (median 15\%; I.Q.R. 3-45\%) and the other histotypes. A significant statistical positive correlation was found between $\mathrm{AI}$ and $\mathrm{PI}(\mathrm{P}=0.036)$, while no association was found between $\mathrm{AI}$ and hENT1 expression, $\mathrm{T}$ factor, $\mathrm{N}$ factor or differentiation grade.

\section{Discussion}

In the present study, we have demonstrated that intestinal-type carcinoma of the ampulla of Vater displays significantly higher levels of hENT1 expression and higher PI than pancreaticobiliary type. Furthermore, a positive correlation between $\mathrm{PI}$ and $\mathrm{AI}$ was also evident. The relationship between apoptosis and cell proliferation, also reported in various neo-

Table 2. Differences in T, N and Grade factors among ampullary subtypes.

\begin{tabular}{lccccccccccccc} 
Histotype & \multicolumn{4}{c}{ T factor } & P* & \multicolumn{3}{c}{ N factor } & P** & \multicolumn{3}{c}{ Grade } & P* \\
& T1 & T2 & T3 & T4 & & N0 & N1 & & 1 & 2 & 3 & \\
Intestinal & 5 & 9 & 6 & 0 & 0.256 & 12 & 8 & 0.821 & 8 & 11 & 1 & 0.007 \\
Pancreaticobiliary & 2 & 5 & 5 & 2 & & 7 & 7 & & 5 & 8 & 1 & \\
\hline Unusual & 0 & 3 & 4 & 0 & & 4 & 3 & & 0 & 2 & 5 & \\
\hline
\end{tabular}

*Kruskal-Wallis test.
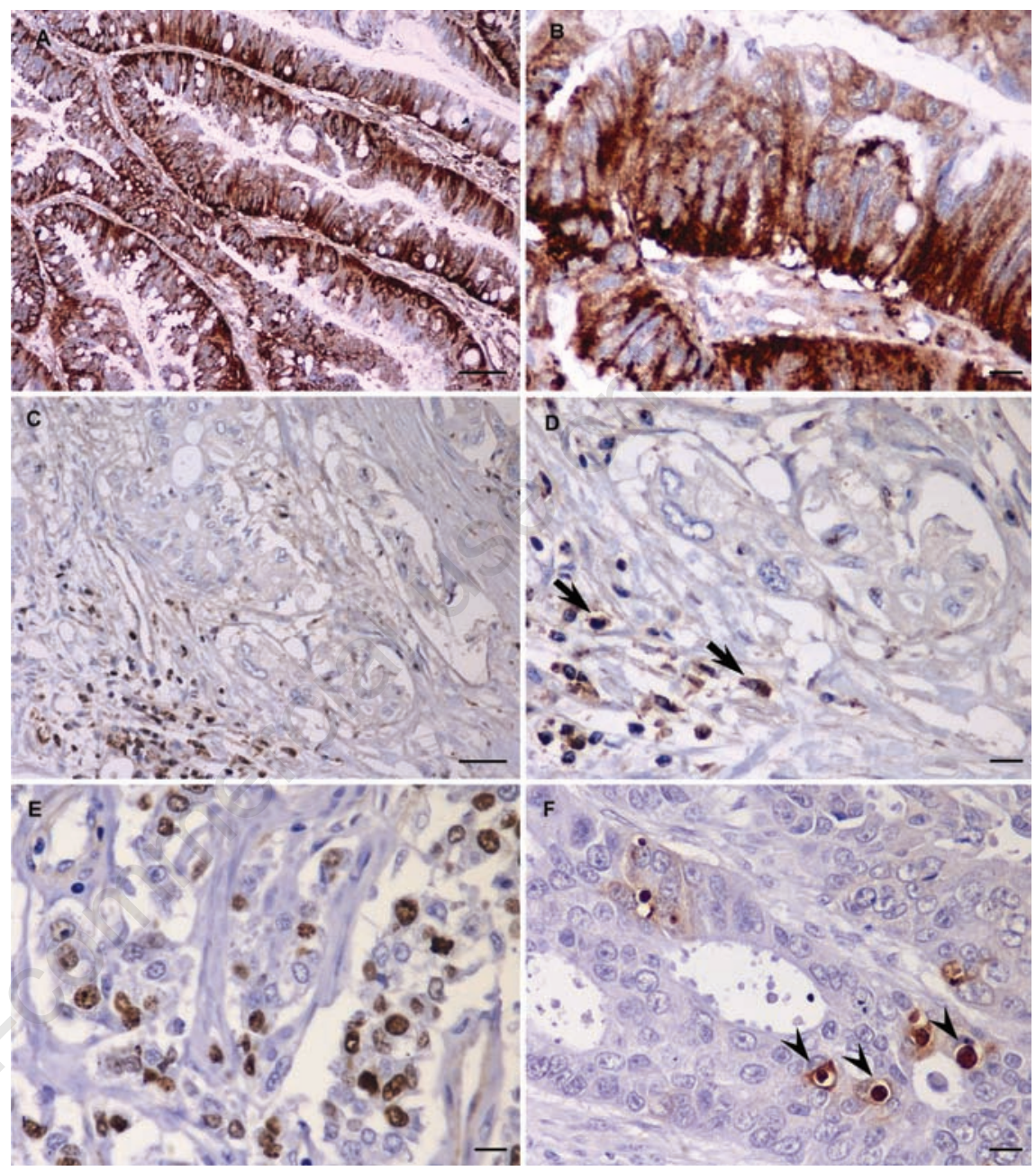

Figure 1. Immunohistochemical staining of ampullary adenocarcinoma. (A) strong immunostaining for hENT1 in intestinal type carcinoma. (B) Details of (A). (C) Negative expression for hENT1 in pancreaticobiliary type carcinoma whereas adjacent lymphocytes (arrows) demonstrate staining and provide a positive internal control. (D) Details of (C). (E) Only distinctly Ki67 (MIB-1) immunoreactive tumour cell nuclei were valued as positive. (F) Strict criteria were used to define a cell as apoptotic: only positive TUNEL stained with the presence of a condensed and often fragmented nucleus together with a surrounding halo (arrowheads). Scale bars: A, C = 50 $\mu \mathrm{m} ; \mathrm{B}, \mathrm{D}, \mathrm{E}, \mathrm{F}=10 \mu \mathrm{m}$.

plasms, suggests a compensatory mechanisms for these events (i.e., the higher number of proliferating cells is reduced by higher levels of apoptosis). ${ }^{20-23}$ However, although PI is closely correlated to AI and hENT1 expression is correlated to higher $\mathrm{PI},{ }^{16}$ no association was found between hENT1 and AI. As a working hypothesis, we propose that hENT1, by allowing nucle-
Table 3. Differences in hENT1 expression among the histotypes.*

Intestinal Pancreaticobiliary

\section{Pancreaticobiliary $\mathrm{P}=0.03$}

\begin{tabular}{lll} 
Unusual & $\mathrm{P}=0.006$ & $\mathrm{P}=0.361$ \\
\hline
\end{tabular}

*Mann-Whitney test. 
osides to enter the cells through the plasma membrane, controls DNA synthesis and therefore proliferation activity, without directly affecting apoptotic mechanisms. Following this hypothesis, our data suggest that hENT-1 expression may generate an imbalance between proliferation and apoptosis (e.g., stimulating proliferation while not affecting apoptosis), playing an important role in the growth of tumour bulk.

Recent studies have suggested that different histological types of ampullary carcinomas may reflect a different biological behaviour. For example, K-Ras mutations were significantly more frequent in intestinal than in the pancreaticobiliary types, and intestinal type adenocarcinomas showed p53 abnormalities more commonly than the pancreaticobiliary type. ${ }^{24}$ Moreover, mucin 2 glycoprotein and cytokeratin (CK) 20 are more intensely expressed in intestinal than in pancreaticobiliary type, while CK7 is more expressed in pancreaticobiliary than intestinal type. ${ }^{25}$ Recently, our group demonstrated that COX-2 protein is significantly more expressed in intestinal than pancreaticobiliary type ampullary cancer. ${ }^{26}$ The direct association between hENT1 expression and tumour growth here suggested gives a biological explanation for the relationship between hENT1 and survival. ${ }^{15}$ In these terms, hENT1, by the function of nucleoside transporter, would represent the intrinsic cellular key to tumour growth, being consequently related to worse prognosis in ampullary carcinoma. In conclusion, our results support the concept of histogenetically different types of ampullary carcinomas, providing the rationale for prospective clinical trials aimed at examining the treatment efficacy by classifying ampullary carcinoma using histological subtypes. Moreover, our results suggest the key role of hENT1 in tumour growth of ampullary carcinoma. Characterization of these tumours based on histopathology, immunohistochemistry and molecular pathology may help to elucidate their aetiology, to define different risk profiles, and to program more tailored therapies.

\section{References}

1. Kimura W, Ohtsubo K. Incidence, sites of origin, and immunohistochemical and histochemical characteristics of atypical epithelium and minute carcinoma of the papilla of Vater. Cancer 1988;61:1394-402.

2. Kimura W, Futakawa N, Yamagata S, Wada Y, Kuroda A, Muto T, et al. Different clinicopathologic findings in two histologic types of carcinoma of papilla of Vater. Jpn $\mathbf{J}$ Cancer Res 1994;85:161-6.

3. Fischer HP, Zhou H. Pathogenesis of carcinoma of the papilla of Vater. J Hepatobiliary
Pancreat Surg 2004;11:301-9.

4. Albores-Saavedra J, Henson DE, Klimstra DS. Malignant epithelial tumours of the ampulla. In: Albores-Saavedra J, Henson DE, and Klimstra DS (eds.) Tumors of the gallbladder, extrahepatic bile duct, and ampulla of Vater. Armed Forces Institute of Pathology, Washington, USA 2000 pp 259316.

5. Abbruzzese JL. New applications of gemcitabine and future directions in the management of pancreatic cancer. Cancer 2002;95:941-5.

6. Dingle BH, Rumble RB, Brouwers MC. The role of gemcitabine in the treatment of cholangiocarcinoma and gallbladder cancer: a systematic review. Can J Gastroenterol 2005;19:711-6.

7. Pasetto LM, D'Andrea MR, Falci C, Monfardini S. Gemcitabine in advanced biliary tract cancers. Crit Rev Oncol Hematol 2007;61:230-42.

8. Mackey JR, Mani RS, Selner M, Mowles D, Young JD, Belt JA, et al. Functional nucleoside transporters are required for gemcitabine influx and manifestation of toxicity in cancer cell lines. Cancer Res 1998;58: 4349-57.

9. Baldwin SA, Mackey JR, Cass CE, Young JD. Nucleoside transporters: molecular biology and implications for therapeutic development. Mol Med Today 1999;5:216-24.

10. Mackey JR, Jennings LL, Clarke ML, et al. Immunohistochemical variation of human equilibrative nucleoside transporter 1 protein in primary breast cancers. Clin Cancer Res 2002;8:110-6.

11. Spratlin J, Sangha R, Glubrecht D, Dabbagh L, Young JD, Dumontet C, et al. The absence of human equilibrative nucleoside transporter 1 is associated with reduced survival in patients with gemcitabine-treated pancreas adenocarcinoma. Clin Cancer Res 2004;10:6956-61.

12. Chow L, Lai R, Dabbagh L, Belch A, Young JD, Cass CE, et al. Analysis of human equilibrative nucleoside transporter 1 (hENT1) protein in non-Hodgkin's lymphoma by immunohistochemistry. Mod Pathol 2005; 18:558-64.

13. Sève $P$, Mackey JR, Isaac $S$, Trédan 0 , Souquet PJ, Pérol M, et al. cN-II expression predicts survival in patients receiving gemcitabine for advanced non-small cell lung cancer. Lung Cancer 2005;49:363-70.

14. Damaraju VL, Elwi AN, Hunter C, Carpenter P, Santos C, Barron GM, et al. Localization of broadly selective equilibrative and concentrative nucleoside transporters, hENT1 and hCNT3, in human kidney. Am J Physiol Renal Physiol 2007;293:F200-11.

15. Santini D, Perrone G, Vincenzi B, Lai R, Cass C, Alloni R et al, Human equilibrative nucleoside transporter 1 (hENT1) protein is associated with short survival in resected ampullary cancer. Ann Oncol 2008;19:724-8.

16. Farrell JJ, Elsaleh H, Garcia M, Lai R, Ammar A, Regine WF, et al. Human equilibrative nucleoside transporter 1 levels predict response to gemcitabine in patients with pancreatic cancer. Gastroenterology 2009;136:187-95.

17. Rosai J. Appendix E. In: Rosai J, Ackerman's editors. Surgical Pathology. Edinburgh, UK, 2004 pp. 2953-4.

18. Sobin LH, Wittekind C. TNM Classification of malignant tumours. In: Sobin LH and Wittekind C (eds.) New York, John Wiley \& Sons, 2002.

19. Santini D, Vincenzi B, Tonini G, Scarpa S, Vasaturo F, Malacrino C, et al. Cyclooxygenase-2 overexpression is associated with a poor outcome in resected ampullary cancer patients. Clin Cancer Res 2005;11:3784-9.

20. Baretton GB, Diebold J, Christoforis G, Vogt M, Müller C, Dopfer K, et al. Apoptosis and immunohistochemical bcl-2 expression in colorectal adenomas and carcinomas. Aspects of carcinogenesis and prognostic significance. Cancer 1996;77:255-64.

21. Tatebe S, Ishida M, Kasagi N, Tsujitani S, Kaibara N, Ito H. Apoptosis occurs more frequently in metastatic foci than in primary lesions of human colorectal carcinomas: analysis by terminal-deoxynucleotidyltransferase-mediated dUTP-biotin nick end labeling. Int J Cancer 1996;65:173-7.

22. Ishida M, Gomyo Y, Tatebe S, Ohfuji S, Ito H. Apoptosis in human gastric mucosa, chronic gastritis, dysplasia and carcinoma: analysis by terminal deoxynucleotidyl transferase-mediated dUTP-biotin nick end labelling. Virchows Arch 1996;428:229-35.

23. Kim YH, Lee JH, Chun H, Nam SJ, Lee WY, Song SY, et al. Apoptosis and its correlation with proliferative activity in rectal cancer. $\mathrm{J}$ Surg Oncol 2002;79:236-42

24. Zhao B, Kimura W, Futakawa N, Muto T, Kubota K, Harihara Y, et al. p53 and p21/Waf1 protein expression and K-ras codon 12 mutation in carcinoma of the papilla of Vater. Am J Gastroenterol 1999; 94:2128-34.

25. Zhou H, Schaefer N, Wolff M, Fischer HP. Carcinoma of the ampulla of Vater: comparative histologic/immunohistochemical classification and follow-up. Am J Surg Pathol 2004;28:875-82.

26. Perrone G, Santini D, Zagami M, Vincenzi $\mathrm{B}$, Verzì A, Morini S, et al. COX-2 expression of ampullary carcinoma: correlation with different histotypes and clinicopathological parameters. Virchows Arch 2006;449:334-40. 